\title{
The vacuum energy crisis
}

\author{
Alexander Vilenkin \\ Institute of Cosmology, Department of Physics and Astronomy, \\ Tufts University, Medford, MA 02155, USA
}

\begin{abstract}
The smallness of the vacuum energy density and its near coincidence with the average matter density of the universe are naturally explained by anthropic selection. An alternative explanation, based on the cyclic model of Steinhardt and Turok, does not address the coincidence problem and is therefore less convincing. This article appeared in "Science" (4 May 2006) as a "perspective" for Steinhardt and Turok's paper in the same issue (astro-ph/0605173).
\end{abstract}


One of the stranger consequences of quantum mechanics is that even empty space has energy. The problem of how to calculate this vacuum energy is arguably the most intriguing mystery in theoretical physics. For decades physicists tried to understand why this energy is so small, but no definitive solution has yet been found. In this week's Science Express, Steinhardt and Turok propose a new approach [1].

Vacuum is empty space, but it is far from being "nothing". It is a complicated physical object in which particles like electrons, positrons and photons are being incessantly produced and destroyed by quantum fluctuations. Such virtual particles exist only for a fleeting moment, but their energies combine to endow each cubic centimeter of space with a nonzero energy. This vacuum energy density does not change in time; it is called the cosmological constant and is usually denoted by $\Lambda$. The trouble is that theoretical calculations of $\Lambda$ give ridiculously large numbers, 120 orders of magnitude greater than what is observed. According to Einstein's General Relativity, vacuum energy produces a repulsive gravitational force, and if the energy were so large, its gravity would have instantly blown the universe apart.

It is conceivable that positive vacuum energy contributions from some particle species are compensated by negative contributions from other species, so that the net result is close to zero. But then the compensation must be amazingly precise, up to 120 decimal places. There seems to be no good reason for such a miraculous cancellation. Until recently, the great majority of physicists believed that something so small could only be zero: some hidden symmetry should force exact cancellation of all contributions to the cosmological constant. However, observations of distant supernova explosions in the late 1990's yielded the surprising discovery that the expansion of the universe accelerates with time [2] - a telltale sign of cosmic repulsion caused by a nonzero (positive) cosmological constant.

The observed magnitude of $\Lambda$ has brought about another mystery: its value is roughly twice the average energy density (or, equivalently, mass density) of matter in the universe. This is surprising because the matter and vacuum densities behave very differently with cosmic expansion. The vacuum density remains constant, while the matter density decreases; it was much greater in the past and will be much smaller in the future. Why, then, do we happen to live at the very special epoch when the two densities are so close to one another? This became known as the cosmic coincidence problem.

Both puzzles can be resolved if one is prepared to assume that the cosmological constant 
is not a fixed number, but takes a wide variety of values in remote parts of the universe. In regions where it is much larger than the observed value, its repulsive gravity will be stronger and will prevent matter from clumping into galaxies and stars [3, 4]. Life is not likely to evolve in such regions.

The idea of "anthropic selection" - that certain features of the universe are selected by the requirement that observers should be there to detect them [5] - runs contrary to the physicist's aspiration to derive all constants of nature from first principles. It has been passionately resisted by the physics community, but has recently gained support from both string theory and cosmology. String theory, the most promising candidate for the fundamental theory of nature, predicts a multitude of vacuum states characterized by different values of $\Lambda$ and other "constants". Inflationary cosmology, which now has a substantial observational support, suggests that the universe on the largest scales is in a state of highenergy exponential expansion and is constantly spawning low-energy "bubbles" like ours, with all possible values of the "constants" (see Fig.1). Galaxies and observers exist only in rare bubbles where $\Lambda$ is small and other constants are also appropriately selected. Analysis shows that most of the galaxies are formed in regions where vacuum and matter densities are about the same at the epoch of galaxy formation [6, 7, 8]. Our present time is close to that epoch, and this explains the coincidence [9, 10].

Steinhardt and Turok propose an alternative explanation for the smallness of $\Lambda$. Building on the idea of Abbott [11], they postulate the existence of a long sequence of vacuum states with $\Lambda$ changing in small increments from one state to the next. If the universe starts with a large cosmological constant, its value will be gradually reduced through a sequence of quantum transitions to lower and lower values. Abbott showed that as $\Lambda$ approaches zero, the transitions become increasingly slow, so the universe spends most of the time in the state with the smallest positive $\Lambda$. He found, however, that the descent to small values of $\Lambda$ takes so long that all matter gets completely diluted by the cosmic expansion, and one ends up with an empty universe. To fix this flaw, Steinhardt and Turok combine Abbott's model with the cyclic cosmological scenario [12], in which the universe goes through multiple cycles of expansion and contraction. The high density of matter is regenerated at the start of each cycle, so 'the empty universe problem' is solved. Most of the cycles will occur while the universe is in the lowest- $\Lambda$ state, and Steinhardt and Turok argue that this state is most likely to be observed. 
The cyclic model is still being developed and is not widely accepted. More importantly, although the S\&T proposal may explain the smallness of $\Lambda$, it does not address the cosmic coincidence problem: why should the smallest possible value of $\Lambda$ be comparable to the present matter density of the universe? The anthropic explanation appears, therefore, to be more compelling.

This work was supported in part by the National Science Foundation.

[1] P.J. Steinhardt and N.G. Turok, Science, 4 May 2006.

[2] A.G. Riess et al., Ap.J. 116, 1009 (1998); S. Perlmutter et al., Ap.J. 517, 565 (1999).

[3] S. Weinberg, Phys. Rev. Lett. 59, 2607 (1987).

[4] A.D. Linde, in 300 years of Gravitation, ed. by S.W. Hawking and W. Israel (Cambridge University Press, Cambridge, 1987).

[5] M. Livio and M.J. Rees, Science 309, 1022 (2005).

[6] A. Vilenkin, Phys. Rev. Lett. 74, 864 (1995).

[7] G. Efstathiou, M.N.R.A.S. 274, L73 (1995).

[8] H. Martel, P.R. Shapiro and S. Weinberg, Ap. J. 492, 29 (1998).

[9] J. Garriga, M. Livio and A. Vilenkin, Phys. Rev. D61, 023503 (2000).

[10] S. Bludman, Nucl. Phys. A663, 865 (2000).

[11] L. Abbott, Phys. Lett. B150, 427 (1985).

[12] P.J. Steinhardt and N. Turok, Science 296, 1436 (2002). 